\title{
Firefighter Safety Using IoT
}

\author{
Caroline Jebakumari S ${ }^{\text {a,1 }}$, Aarthi K ${ }^{\text {a }}$, Darshan S a , Karan S a \\ ${ }^{a}$ Department of Electronics and Communication Engineering, Easwari \\ Engineering College, Chennai, TN, India
}

\begin{abstract}
From ancient times, firefighter's faces a high risk of burns which may sometimes lead to death, especially those who enter the burning building first and those who are holding the front end of the nozzle. In order to avoid this kind of risk, an enlightened physical admonition arrangement for firefighter was put forward. Corresponding controller is developed with low power consumption on the display screen terminator which could inspect numerous conflagration in the surroundings and the site where firefighter reside as substantially as their critical bodily data so that the admonition might be provided with a low transmitting error rate. Moreover, the suitable information processing computation is sorted out to assure the preciseness. Consequently, competent firefighter's individual protection is augmented considerably with strong anti-interference. In addition to this, a buzzer will be there on the side of firefighter like alarm when parameters like temperature, blood pressure, heart rate becomes abnormal so that they might not take the risk considering their body conditions.
\end{abstract}

Keywords. Multi-sensor, physical warning, low power consumption, cloud

\section{Introduction}

The difficulty of fire control became hopeless as the firefighter uniform were somewhat retrogressive as substantially as the shutdown edifice are augmented at present. The conflagration control became recalcitrant even in recent times as two firemen lost their lives while fighting the flames in the Madurai textile shop. In order to reduce this kind of incidents this system has been preferred. The Fire department uniform has to be upgraded as this was crucial to assure the firefighter protection, which has been examined in the earlier instance. Thus, an enlightened physical caution scheme for firefighter premised on IoT has been envisaged. The physiological information of the fireman and the site where the fireman remains will be collected by this system and will be forwarded to display screen via cordless procedure. Omni bearing oversees task for the firefighter individual protection, preferable protection factor and servicing execution are accomplished by utilizing this network. As considerably as such method might arrive at the worldwide sophisticated tier to the point of a firefighter functioning orderliness and existence aid. While integrating sensing element in a firefighter's uniform, the conflagration surroundings and the site might be recognized consequently the ones might evade damage to the utmost. In order to give a detailed study about the controllers, this paper has been organized into the following sections.

- Segment 1 set out the introduction for this proposed methodology and it describes the related works which deal with various types of sensors and its

\footnotetext{
${ }^{1}$ Caroline Jebakumari S, Department of Electronics and Communication Engineering, Easwari Engineering College, Chennai, TN, India; Email: caroline.s@eec.srmrmp.edu.in.
} 
advantages and disadvantages compared to the previous designs and performance.

- Segment 2 describes the proposed methodology and its advantages.

- Segment 3 characterizes outcome and examination of the projected system.

- Segment 4 account termination and prospect work for fire fighter's safety using IoT.

Existing System

T. Brunschwiler [1] proposed a system which measures the pressure in firefighter while learning in heated workout locale and provided a task to obtain specialist mark. It is also concentrated on Chronic-Pulmonary-Disease. While this system measures the stress in firemen which is the major advantage but still there are many disadvantages this system hasn't proposed which includes physiological parameters when the firefighter is in danger.

Sarita Gupta [2] insists that fire is the major cause for accidental in this World so a system was proposed that were destined to live up to consumer guideline that has a micro - controller, RF modules and two types of sensors [7]. If a flame were ascertained, the information and Uniform resource locator address of damaged locale is forwarded to the conflagration supervision department via The Global System for Mobile Communications unit and without delay the salvage functioning will take place. The above technology does not reveal the nature or physiological parameters of firefighter.

\section{Proposed System}

Temperature spotting, pulse oximeter, concentration of carbon monoxide spotting and IoT module are the physiological confluence that this paper reflects on as dominant core [3]. The warning system keeps track of a firefighter's physical environment in real time through a focal point confluence the particulars are communicated to display screen fumigator. Apart from the earlier points, cloud keeps track of a firefighter's physiological conditions and the surroundings where firefighter remains. The latitude and longitude where the firefighter stays will also be monitored and when there is any abnormal state in any one of the physiological activities, the person from the outside will go for rescue operation and there will be a buzzer insisting that the firefighter is in danger, so that the firefighter gets indicated about his physiological activities. The algorithm that this system follows, advantages that this system possesses and the components used are explained below.

\subsection{Fire Fighter Algorithm}

The steps specified below is the algorithm for this proposed system,

- The Firefighter who is inside the building or a place which is on fire will be having different kinds of sensors inside firefighter's suit in order to monitor different body parameters.

- The values from the sensor will be updated in a cycle at a certain period of time in an URL link. The one who is monitoring from outside can monitor those values.

- The temperature sensor (LM35) has been set to a limit of $40^{\circ} \mathrm{C}$. When the surrounding temperature of the firefighter is below $40^{\circ} \mathrm{C}$ it displays as "TEMP_NORMAL" in the URL link. When the surrounding temperature goes above 
$40^{\circ} \mathrm{C}$, it displays as "TEMP_ABNORMAL".

- Gas sensor (MQ-02) will show a value of 1 on the screen and displays "GAS_NORMAL" when there are no harmful gases in the surrounding environment of the firefighter. When it detects any harmful gas, then it displays "GAS_ABNORMAL" and the value changes to 0 .

- Pulse Oximeter (MAX30100) displays "HB_NORMAL" when the heart rate $>10$ and displays "HB_ABNORMAL" when the heart rate $<10$. Blood pressure sensor (Mercury sphygmomanometer), displays "BP_NORMAL" when the blood pressure $>$ 80 and displays "BP_ABNORMAL" when blood pressure $<80$.

- The locale of the firefighter gets updated in the URL link at a certain interval of time. It displays location as latitude and longitude coordinates in Figure 1. All the sensor values which are mentioned in the condition can be changed and can be used for different circumstances. While showing these values in a table the date and time gets updated.

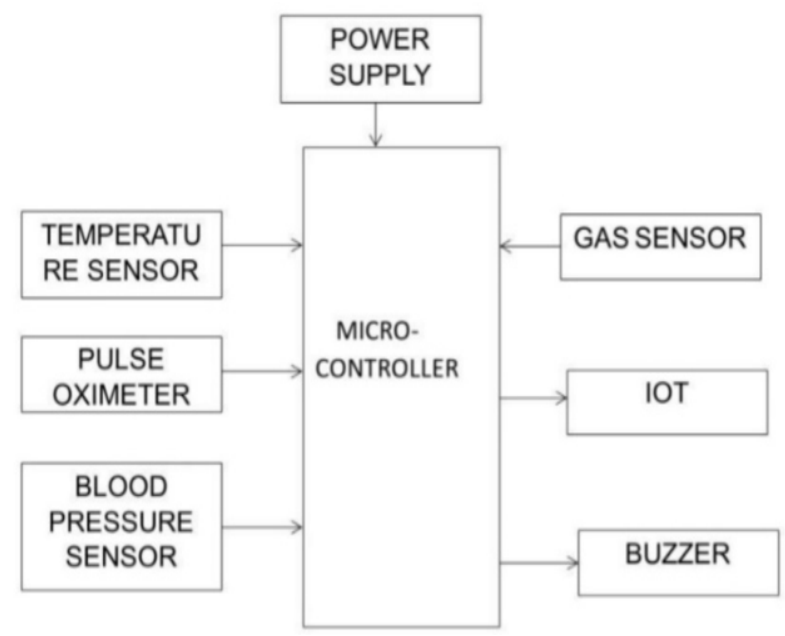

Figure 1. Block Diagram of Proposed System

\subsection{Advantages}

The advantages that this paper hold are when the firefighters in scorch forests, it is not easy to know the location when he is in the hazard [4]. Using this system, the locale can be easily found, so that someone from the external can salvage the firefighters who are in peril. When deleterious gases like Carbon monoxide or some other gases is sensed via gas sensor, then it will be updated to the person external. So that later on, no one will get in that spot or will walk inside with some indispensable pre-emptive actions [5]. When pernicious gases surround the fire fighters or when the encircling temperature goes very severe the person who is overseeing from the outside will know that, but to specify that to the firefighter inside, a buzzer is kept within the firefighter [6].

\subsection{Components Used}

The components used here are power supply, temperature sensor (LM35), blood pressure sensor, gas sensor (MQ-02), pulse oximeter (MAX30100), as it operates from 
$4 \mathrm{~V}$ to $30 \mathrm{~V}$, the scale factor for $\mathrm{LM} 35$ is $0.5^{\circ} \mathrm{C}$ and ranges from $-55^{\circ} \mathrm{C}$ to $150^{\circ} \mathrm{C}$. ATmega328P can be used in real time bio-metrics, robotics and academic applications. MAX30100 is a well conceived pulse oximeter sensor. Mercury sphygmomanometer being used for measuring blood pressure sensor. MQ-02 is the best for gas sensor as it detected the hydrogen gas leakage and portable gas.

\section{Results and Analysis}

While the power supply is given, the ARDUINO board shows the HB, G, BT, BP where HB indicates the Heartbeat value, $G$ represent the gas sensor value, BT represents the temperature value, BP represents the blood pressure value. According to the situation the value varies. Initial values that are specified in the controller in Figure 2.

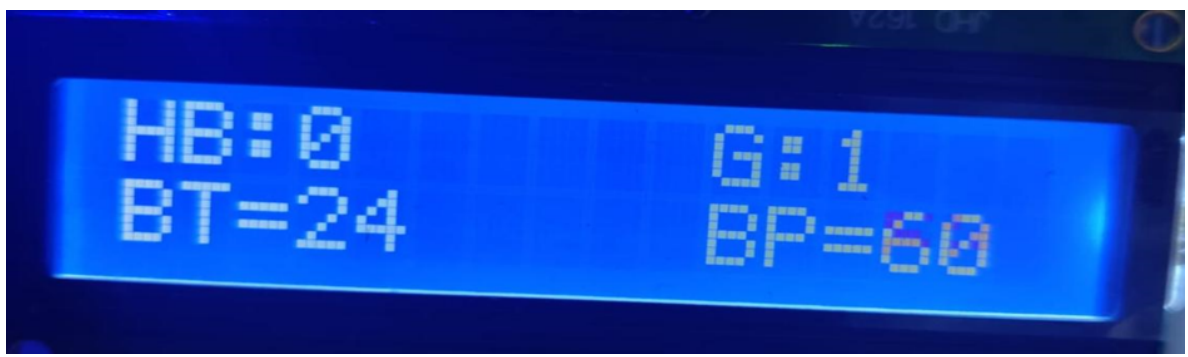

Figure 2. Display image of the micro-controller.

Figure 3 shows the updated sensor values of the system. The Table 1 has the following entries which show whether temperature, gas, pulse rate; blood pressure is normal or abnormal for the fire fighter. In case of any abnormal condition sensor 5 in the Table 1 indicates an EMERGENCY situation so that the person who is monitoring from the external will get alerted. Here, the Date \& Time will also be pointed out while the sensor values are getting processed.

Table 1. Displays the physiological nature of the fire fighter

\begin{tabular}{|c|c|c|c|c|c|c|c|c|c|}
\hline$\# 1$ & Sensor 1 & Sensor 2 & Sensor 3 & Sensor 4 & Sensor 5 & $\begin{array}{l}\text { Sensor } \\
6\end{array}$ & $\begin{array}{l}\text { Sensor } \\
7\end{array}$ & $\begin{array}{l}\text { Sensor } \\
8\end{array}$ & $\begin{array}{l}\text { Date } \\
\text { \& } \\
\text { Time }\end{array}$ \\
\hline 1 & TEMP_NORMAL & GAS_NORMAL & HB_ABNORMAL & BP_ABNORMAL & EMERGENCY & null & null & null & $\begin{array}{l}2021-03- \\
05 \\
13: 33: 45\end{array}$ \\
\hline 2 & TEMP_NORMAL & GAS_NORMAL & HB_ABNORMAL. & BP_ABNORMAL & EMERGENCY & null & null & null & $\begin{array}{l}\text { 2021-03- } \\
05 \\
\text { 13:33:14 }\end{array}$ \\
\hline 3 & TEMP_NORMAL & GAS_NORMAL & HB_ABNORMAL & BP_ABNORMAL & EMERGENCY & null & null & null & $\begin{array}{l}2021-03- \\
05 \\
13: 31: 35\end{array}$ \\
\hline 4 & TEMP_NORMAL & GAS_NORMAL & HB_ABNORMAL & BP.ABNORMAL & EMERGENCY & null & null & null & $\begin{array}{l}2021-03- \\
05 \\
13: 30: 41\end{array}$ \\
\hline 5 & TEMP_NORMAL & GAS_NORMAL & HB_ABNORMAL & BP,ABNORMAL & EMERGENCY & null & null & null & $\begin{array}{l}2021-03- \\
05 \\
13: 29: 43\end{array}$ \\
\hline
\end{tabular}


Latitude and longitude of the firefighter are laid out in the Table 2, so that the local of the firefighter can be easily found when the firefighter is in peril.

Table 2. Displays the latitude and longitude along with date and time

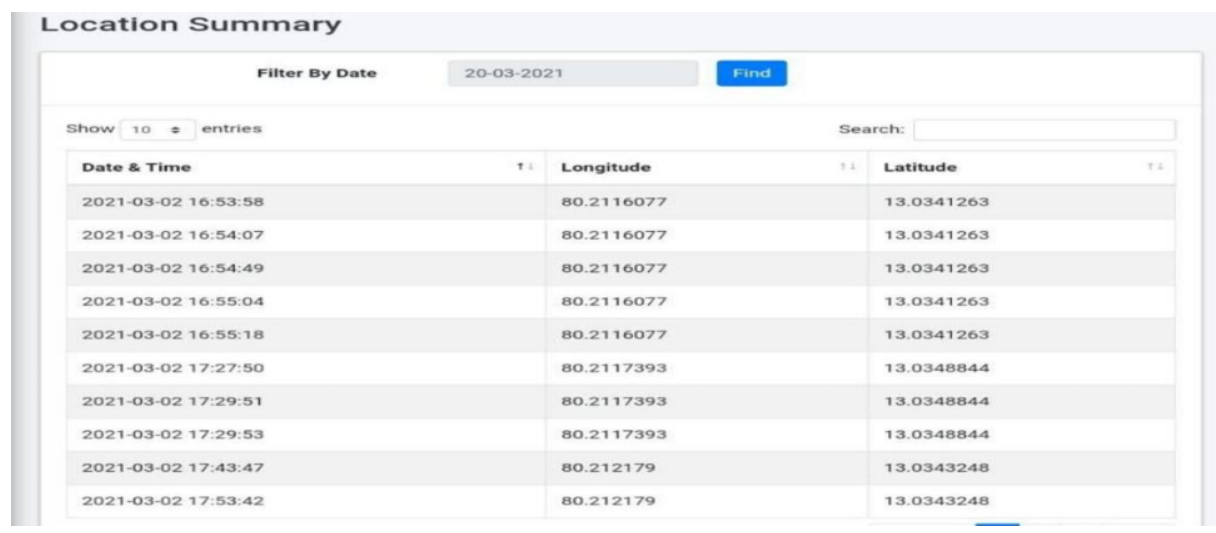

Finally, the complete hardware setup of the proposed system is shown in Figure 3. The complete setup which includes power supply adapter, power supply, Arduino board, temperature sensor (LM35), blood pressure sensor, pulse oximeter, gas sensor, IoT and a buzzer. Here, the result of the proposed system is discussed and analyzed.

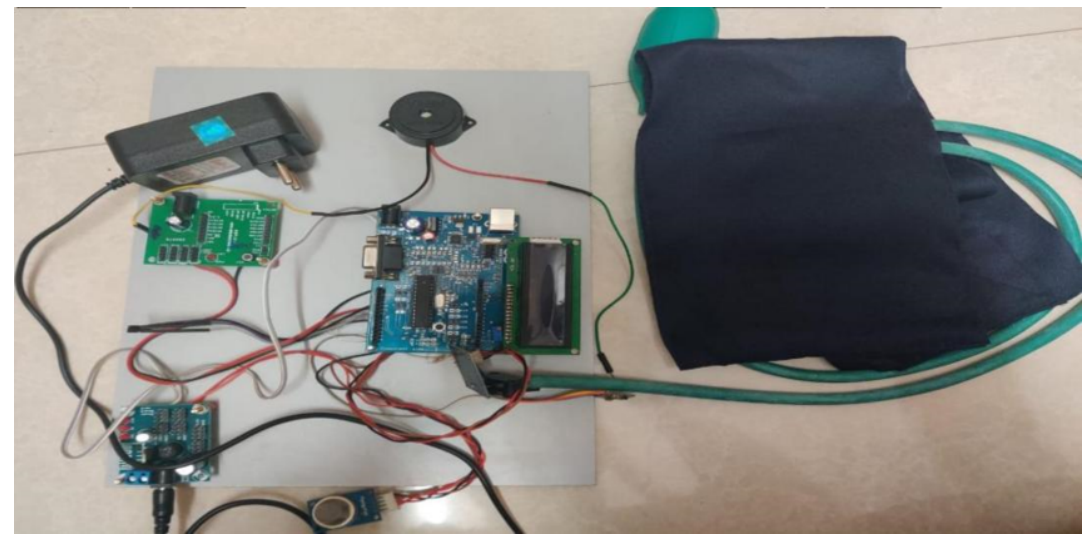

Figure 3. Project Kit Image

\section{Conclusion}

The physical warning system for firefighter is done in this paper with less network technology, IOT, sensor, embedded and computer technology to produce this intelligent system. It collects information from the firefighter like temperature, blood pressure, pulse and the gas which firefighter is inhaled and according to the values the buzzer rings to the firefighter simultaneously the values are shown to the one who is monitoring from outside those values will be saved in an URL address. The result of this system has many edges which will help the firefighter who are working in a dangerous position like building, forest and quarries. Thus, an equipment for firefighter 
safety using IOT has been done to protect the firefighter who are in danger. In the future, GPS which is in-built at present can be improved like even when there are many floors in buildings in which floor or in which room the firefighter is present can be found. The sensors can also be covered with thick sheet or metal to withstand more temperature. These are the expected improvisation in the future for the proposed system.

\section{References}

[1] Hackett, S., Cai, Y., \& Siegel, M. (2019). Activity Recognition from Sensor Fusion on Fireman's Helmet. 2019 12th International Congress on Image and Signal Processing, BioMedical Engineering and Informatics (CISP-BMEI), 1-6. https://doi.org/10.1109/CISP-BMEI48845.2019.8966005

[2] Brunschwiler, T., Weiss, J., Paredes, S., Sridhar, A., Pluntke, U., Chau, S. M., Gerke, S., Barroso, J., Loertscher, E., Temiz, Y., Ruch, P., Michel, B., Zafar, S., \& van Kessel, T. (2019). Internet of the Body - Wearable Monitoring and Coaching. 2019 Global IoT Summit (GIoTS), 1-6. https://doi.org/10.1109/GIOTS.2019.8766409

[3] Gupta, S., Mudgil, A., Bhardwaj, P., \& Gupta, M. (2016). Design and Development of Automatic Fire Alert System. 2016 8th International Conference on Computational Intelligence and Communication Networks (CICN), 632-636. https://doi.org/10.1109/CICN.2016.130

[4] N. Brushlinsky, M. Ahrens, S. V. Socolow, P. Wagner, "World Fire Statistics. Technical report 24" Centre of Fire Statistics Int. Assoc. of Fire and Rescue Services, 2019.

[5] Jiang, W., Chen, C., \& Cai, Y. (2016). The design on intelligent physical warning system for fireman. 2016 International Conference on Audio, Language and Image Processing (ICALIP), 694-698. https://doi.org/10.1109/ICALIP.2016.7846531

[6] R. G. Babu, A. Nedumaran and A. Sisay, "Machine Learning in IoT Security Performance Analysis of Outage Probability of link selection for Cognitive Networks," 2019 Third International conference on I-SMAC (IoT in Social, Mobile, Analytics and Cloud) (I-SMAC), 2019, pp. 15-19, https://doi.org/ 10.1109/I-SMAC47947.2019.9032669

[7] R. G. Babu, V. V. Nathan, J. Bino, C. Amali and S. Ganesh, "IoT Security Enhancement with Automated Identification Device using IOT SENTINEL," 2021 11th International Conference on Cloud Computing, Data Science \& Engineering (Confluence), 2021, pp. 518-523, https://doi.org/10.1109/Confluence51648.2021.9377165 